\title{
Gingival crevicular fluid levels of cytokine, chemokine, and growth factors in patients with periodontitis or gingivitis and periodontally healthy subjects: a cross-sectional multiplex study
}

\author{
HASAN GÜNDOĞAR ${ }^{1,2,3}$, KEMAL ÜSTÜN ${ }^{4}$, SÜLEYMAN ZIYA ŞENYURT ${ }^{3}$, EDA ÇETIN ÖZDEMIR \\ UFUK SEZER ${ }^{6}$, KAMILE ERCIYAS
}

${ }^{1}$ Department of Surgery and Pediatric Dentistry, Khoja Akhmet Yassawi International Kazakh-Turkish University, Turkestan, Kazakhstan ${ }^{2}$ Department of Medical Biochemistry, Faculty of Medicine, University of Gaziantep, Gaziantep, Turkey

${ }^{3}$ Department of Periodontology, Faculty of Dentistry, Gaziantep University, Turkey

${ }^{4}$ Department of Periodontology, Faculty of Dentistry, Akdeniz University, Turkey

${ }^{5}$ Department of Periodontology, Faculty of Dentistry, Kahramanmaraş Sütçü İmam University, Turkey

${ }^{6}$ Department of Periodontology, Faculty of Dentistry, İstanbul Gelişim University, Turkey

\begin{abstract}
Introduction: Gingival crevicular fluid $(G C F)$ is an important resource for detecting inflammatory biomarkers related to periodontal disease. The purpose of this research was to identify the possible relation between cytokine levels and periodontal status.

Material and methods: GCF samples collected from 25 periodontally healthy individuals, 24 with gingivitis, and 24 with periodontitis were analyzed using the MAGPIX system with a Bio-Plex Pro Human Cytokine 27-plex kit. Gingival index (GI), periodontal pocket depth (PPD), plaque index (PI), and clinical attachment level (CAL) were measured from each tooth to determine each patient's periodontal status.

Results: All clinical parameters showed statistically significant differences between groups. While interleukin (IL)-1 $\beta$, IL-6, macrophage chemotactic protein 1 (MCP-1), interferon $\gamma$-induced protein 10 (IP-10) and vascular endothelial growth factor (VEGF) levels were statistically significantly higher in patients with periodontitis compared to periodontally healthy subjects, IL-15 levels were found to be statistically significantly higher in periodontally healthy individuals compared to periodontitis $(p<0.05)$. Also, IL-1 $\beta$ and IP-10 showed positive correlations with PPD, CAL, GI and bleeding on probing (BOP).

Conclusions: It is considered that IL-1 $\beta, I L-6, I L-15, M C P-1$, VEGF and IP-10 could be related to periodontal disease and health and can be used as an adjunct to clinical examination in future research design. Scanning cytokines in GCF with a multiplex immunoassay technique is consequential, especially because many cytokine types are found in one sample.
\end{abstract}

Key words: periodontitis, gingivitis, periodontal health, laser doppler flow cytometry.

(Cent Eur J Immunol 2021; 46 (4): 474-480)

\section{Introduction}

Periodontal diseases are inflammatory diseases that can cause loss of tooth and tooth supporting tissue, which are the result of specific and complex interactions between pathogenic bacteria in the dental plaque and host tissue [1]. Gingivitis and periodontitis are the most common periodontal diseases. Although previous studies showed the virulence of periodonto-pathogen bacteria, the existence of microbial dental plaque alone is not enough for the development of periodontitis [2]. Moreover, in some studies, the existence of periodonto-pathogens in periodontally healthy areas has also been observed [3]. In other words, while the existence of dental plaque or biofilm is a necessary factor for the beginning of periodontal diseases, the progression of the disease and alveolar loss are related not only to the microbiota but also to the host response [4]. Biological mediators such as cytokines and growth factors may reflect and show the host response capacity [5].

Cytokines are important mediators that play roles in various significant biological activities, such as early inflammation and proliferation, regeneration, differentiation, 
and hemostasis, and they are also secreted from various inflammatory cells. In the formation and progression of periodontal diseases, cytokines have important roles to play, and most of these cytokines have been detected in gingival crevicular fluid (GCF). Furthermore, growth factors such as cytokines are mediators that play important roles in cell regeneration and differentiation [6]. Both cytokines and growth factors are collected simply from GCF and used for clinical research and epidemiological studies [4]. Moreover, there are many innovative challenges in periodontology for enhancement of the diagnostic and prognostic potential, especially diagnostic. Identifying biomarkers in GCF is the most important innovative approach for improving the capacity for diagnosis and prognosis. Numerous biomarkers are identified in GCF. Among these biomarkers, cytokines in GCF have an important place [7].

Clinical examination is one of the most important diagnostic tools for periodontal disease. The assessment of clinical measurements and radiological examinations ordinarily ensures information about the periodontal destruction background, the current status of periodontal disease, and the prognosis [8]. However, some studies have revealed cytokines such as interleukin (IL)-1 $\beta$, IL-6, tumor necrosis factor $\alpha$ (TNF- $\alpha$ ), IL-4, IL-10, etc., in systemically healthy patients [9] and also in patients with systemic disease [10]. Moreover, previous studies showed that some cytokine and growth factors, especially IL-1 $\beta$, were related to periodontitis [7].

All inflammatory cells in the periodontally diseased area and cytokines and growth factors secreted from these cells behave in harmony. Although the level of gingival crevicular fluid and changes in cytokine and growth factor levels in periodontal disease have been studied previously, almost none of these studies could determine the relationship of the cytokines with each other, and also usually they did not have a gingivitis group. The present study was aimed to comparatively analyze the level of cytokines and growth factors in GCF, and also compared with each other in periodontally healthy individuals as well as gingivitis and periodontitis patients.

\section{Material and methods}

\section{Study population and design}

This research was designed as a single-blind, cross-sectional study and was conducted with 25 periodontally healthy individuals and 24 gingivitis and 24 chronic periodontitis patients referred to the Department of Periodontology Clinic in the Faculty of Dentistry at Gaziantep University with different periodontal complaints. Although periodontally healthy, gingivitis and chronic periodontitis criteria were based on the 1999 American Academy of Periodontology [11], according to prior periodontal examination and radiographical records, they were changed from 1999 to 2018 European Federation of Periodontology workshop criteria [12]. All individuals provided written informed consent, and the study protocol was approved by the Ethics Committee of Gaziantep University, Gaziantep, Turkey (Number: 168, Date: 16.04.2013).

\section{Inclusion and exclusion criteria}

The inclusion criteria for the study were that: individuals should be systemically healthy, not being pregnant or within lactation periods, not using any medication that could affect periodontal tissues such as beta blockers, not having used any antibiotics or nonsteroidal inflammatory medication in the previous 6 months, and not smokers or drinkers (never started or stopped at least 10 years ago). Locally, patients have at least 18 teeth.

Patients with local periapical pathologies, caries or restorations according to periapical or panoramic X-ray examination were not included in the study. In addition, if there was a history of orthodontic and periodontal treatment (surgical or non-surgical), they were not included in the study.

\section{Clinical examination and radiological examination}

All clinical measurements were examined by a single intra-caliber clinician (H.G.). Measurements were recorded in a specially prepared chart form on a computer (Microsoft Office, Excel, USA). For these measurements, a Williams-type periodontal probe (Hu-Friedy, Chicago, IL, USA) with a $0.5-\mathrm{mm}$ diameter was used. The evaluated clinical parameters were determined as probing pocket depth (PPD), clinical attachment level (CAL), plaque index (PI), gingival index (GI) and bleeding on probing (BOP). All clinical parameters were recorded on six different sides of all teeth (mesiobuccal, midbuccal, distobuccal, mesioligual/palatinal, midlingual/palatinal, and distolingual/ palatinal). For the periodontally healthy group total BOP was adjusted below $10 \%$ and for the gingivitis group $30 \%$.

Overall all, based on the 1999 classification, the groups were: periodontally healthy $(H)$, gingivitis $(G)$ and chronic periodontitis $(\mathrm{P})$. Only $\mathrm{P}$ groups changed based on the 2018 classification. The $\mathrm{P}$ groups have 8 patients in stage 3 and the others in stage 2 .

\section{Gingival crevicular fluid sample collection and sample analysis}

Gingival crevicular fluid samples were collected from all patients at particular hours of the day and before periodontal measurements were performed. Areas selected for each tooth were isolated with cotton rolls in order to prevent contamination by saliva. After supragingival plaque was carefully removed, it was dried with air spray, absorptive sterile prefabricated paper strips (Periopaper, Orafow Inc., Plainview, New York, USA) were slowly inserted into the periodontal pocket until a gentle pressure was felt and were kept inside the pocket for 30 seconds. 
During this procedure, mechanical irritation was avoided. The GCF volumes of samples were measured with a calibrated Periotron 8000 (Periopaper, OraFlow Inc., Plainview, New York, USA) device. The samples, which had been placed in Eppendorf tubes, were kept at $-80^{\circ} \mathrm{C}$ until analysis. The analysis of GCF samples was carried out in the Institute of Medical Oncology laboratory in the Faculty of Medicine at Dokuz Eylül University with a Bio-Plex MAGPIX Multiplex Reader device using Bio-Plex 27 plex cytokine immunoassay kits (Bio-Rad, USA) [IL-1 $\beta$, IL-1ra, IL-2, IL-4, IL-5, IL-6, IL-7, IL-8, IL-9, IL-10, IL-12 (p70), IL-13, IL-15, IL-17, fibroblast growth factor (FGF), eotaxin, granulocyte colony-stimulating factor (G-CSF), granulocyte-macrophage colony-stimulating factor (GM-CSF), interferon $\gamma$ (IFN- $\gamma$ ), interferon $\gamma$-induced protein 10 (IP-10), macrophage chemotactic protein 1 (MCP-1), macrophage inflammatory protein (MIP)-1 $\alpha$, MIP-1 $\beta$, platelet-derived growth factor (PDGF)-BB, RANTES, TNF- $\alpha$, and vascular endothelial growth factor (VEGF)] in accordance with Teles et al. [8].

\section{Statistical analysis}

For the analysis of the data obtained in our study, SPSS for Mac version 22.0 software was used and the data were accepted as statistically significant at $p<0.05$. The normality of numerical data was tested by the Shapiro-Wilk test. In the intergroup comparison of non-normal distributed variables, the Mann-Whitney $U$ test was used, while ANOVA and Tukey comparison tests were used to compare normally distributed numerical data. Correlations between clinical and biochemical variables were tested with Spearman rank correlation coefficient. The average statistics are given as mean \pm standard deviation for descriptive statistics.

\section{Results}

Systemically healthy and non-smoker 24 periodontitis, 24 gingivitis patients, and 25 periodontally healthy individuals aged between 20 and 58 years old $(40.8 \pm 8.45)$ were included in the research (Table 1).

\section{Clinical finding}

Although PPD, CAL and GI showed statistically significant differences when comparing all groups with each other, and PI showed statistically significant differences between periodontally healthy subjects and diseased patients, there were no statistically significant differences between gingivitis and periodontitis groups (Table 2).

\section{Biochemical findings}

In terms of the GCF levels of biochemical parameters with intergroup comparison, IL-1 $\beta$, IL-6, MCP-1, IP-10, and VEGF levels were found to be significantly higher in periodontitis patients compared to periodontally healthy individuals. Interleukin 15 levels showed statistically significant differences between healthy individuals and patients with periodontitis. However, other cytokine and chemokine values did not show any statistically significant differences in the intergroup comparison. Also, the gingivitis group showed no statistically significant differences compared with healthy and periodontitis groups (Table 3).

\section{Correlations}

The correlations between clinical parameters (PPD, CAL, GI, PI and BOP) and biochemical parameters are shown at Table 4. Although FGF, G-CSF, IFN- $\gamma$, IL-10, IL-12, IL-17, IL-1ra, IL-4, IL-5, IL-6, IL-7, IL-9, MCP-1, MIP- $1 \alpha$, PDGF, RANTES, TNF- $\alpha$ and VEGF did not

Table 1. Distributions of age and gender

\begin{tabular}{|c|c|c|c|c|c|c|}
\hline \multirow[t]{2}{*}{ Parameters } & \multicolumn{2}{|c|}{ Periodontally healthy } & \multicolumn{2}{|c|}{ Gingivitis } & \multicolumn{2}{|c|}{ Periodontitis } \\
\hline & Min. & Max. & Min. & Max. & Min. & Max. \\
\hline Age & 20 & 47 & 24 & 52 & 32 & 58 \\
\hline \multirow[t]{2}{*}{ Gender } & Male & Female & Male & Female & Male & Female \\
\hline & 11 & 14 & 13 & 11 & 12 & 12 \\
\hline
\end{tabular}

Table 2. Comparison of clinical parameters

\begin{tabular}{|c|c|c|c|c|c|c|}
\hline \multirow{2}{*}{$\begin{array}{l}\text { Clinical } \\
\text { parameters }\end{array}$} & \multicolumn{2}{|c|}{ Periodontally healthy } & \multicolumn{2}{|c|}{ Gingivitis } & \multicolumn{2}{|c|}{ Periodontitis } \\
\hline & Mean & SD & Mean & SD & Mean & SD \\
\hline PPD (mm) & $1.08^{\dagger}$ & 0.27 & $1.96^{*}$ & 0.62 & $5.42 *, \dagger$ & 0.83 \\
\hline CAL (mm) & $1.87^{\dagger}$ & 0.07 & $2.39 *$ & 0.23 & $6.27 *, \dagger$ & 1.20 \\
\hline PI & $1.26^{\dagger}$ & 0.15 & $2.07 *$ & 0.20 & $2.41 *$ & 0.42 \\
\hline GI & $1.04^{\dagger}$ & 0.03 & $1.57 *$ & 0.11 & $1.85^{*, \dagger}$ & 0.24 \\
\hline
\end{tabular}


Table 3. Comparison of biochemical parameters

\begin{tabular}{|c|c|c|c|c|c|c|}
\hline \multirow{2}{*}{$\begin{array}{l}\text { Biochemical } \\
\text { parameters }\end{array}$} & \multicolumn{2}{|c|}{ Periodontally healthy } & \multicolumn{2}{|c|}{ Gingivitis } & \multicolumn{2}{|c|}{ Periodontitis } \\
\hline & Mean & SD & Mean & SD & Mean & SD \\
\hline IL-1 $\beta$ & 21.73 & 17.53 & 44.63 & 40.63 & $61.15^{*}$ & 55.24 \\
\hline IL-1ra & 6.12 & 3.54 & 5.71 & 2.10 & 4.91 & 2.02 \\
\hline IL-2 & 11.13 & 10.69 & 14.92 & 28.19 & 8.73 & 7.19 \\
\hline IL-4 & 3.53 & 1.68 & 3.64 & 1.17 & 4.38 & 2.25 \\
\hline IL-5 & 1.44 & 0.71 & 1.49 & 0.68 & 3.62 & 8.76 \\
\hline IL-6 & 7.03 & 3.90 & 12.60 & 13.51 & $17.59^{*}$ & 37.66 \\
\hline IL-7 & 4.16 & 3.92 & 3.63 & 1.40 & 4.92 & 4.61 \\
\hline IL-8 & 137.33 & 125.88 & 94.64 & 126.95 & 93.13 & 89.84 \\
\hline IL-9 & 2.22 & 0.87 & 2.07 & 1.00 & 2.41 & 1.10 \\
\hline IL-10 & 75.35 & 55.99 & 79.97 & 34.37 & 69.79 & 35.98 \\
\hline IL-12 & 51.43 & 65.18 & 48.24 & 26.70 & 50.69 & 28.77 \\
\hline IL-13 & 37.37 & 23.86 & 28.71 & 18.16 & 20.93 & 14.14 \\
\hline IL-15 & 12.36 & 6.44 & 10.51 & 7.26 & $6.96 *$ & 4.52 \\
\hline IL-17 & 2.89 & 1.39 & 3.05 & 2.08 & 3.23 & 1.82 \\
\hline Eotaxin & 12.55 & 31.28 & 7.11 & 2.04 & 6.73 & 2.69 \\
\hline FGF- $\beta$ & 6.66 & 2.43 & 16.86 & 33.74 & 7.87 & 5.04 \\
\hline G-CSF & 5.61 & 7.07 & 4.63 & 4.60 & 5.23 & 4.10 \\
\hline GM-CSF & 14.92 & 7.40 & 13.92 & 6.33 & 11.75 & 7.64 \\
\hline IP-10 & 2.66 & 5.27 & 3.24 & 5.79 & $9.85^{*}$ & 33.11 \\
\hline MIP- $1 \alpha$ & 6.74 & 4.77 & 10.45 & 8.32 & $16.14^{*}$ & 35.36 \\
\hline MIP-1 $1 \beta$ & 152.98 & 117.10 & 357.26 & 639.24 & 126.93 & 135.86 \\
\hline PDGF & 6.10 & 4.01 & 5.27 & 2.89 & 5.68 & 1.85 \\
\hline RANTES & 171.79 & 669.83 & 47.69 & 40.40 & 69.97 & 83.36 \\
\hline TNF- $\alpha$ & 1.50 & 0.73 & 1.74 & 0.50 & 1.98 & 1.08 \\
\hline VEGF & 70.25 & 52.25 & 61.42 & 51.60 & $109.86^{*}$ & 92.94 \\
\hline IFN- $\gamma$ & 3.59 & 2.06 & 3.76 & 1.55 & 3.91 & 1.84 \\
\hline MCP-1 & 5.62 & 3.91 & 8.14 & 7.56 & $10.64^{*}$ & 4.39 \\
\hline
\end{tabular}

show statistically significant correlation with all clinical parameters (PPD, CAL, GI, PI and BOP), MIP-1 $\beta$, IL-2, IL-15 levels in GCF showed statistically significant negative correlations with all clinical parameters. While there were negative correlation between IL- 8 and BOP, IL-1 $\beta$ and IP-10 showed positive correlations with PPD, CAL, GI and BOP. In addition, while there was a negative correlation between IL-13 and CAL, a negative correlation was found between eotaxin and CAL, GI, BOP and also between GM-CSF and CAL, GI, PI, BOP.

\section{Discussion}

Periodontal diseases such as periodontitis and gingivitis are inflammatory diseases that occur after the re- lationship between dental plaque and the host response. The host response delivering biochemical molecules such as cytokines and growth factors in GCF can be found and detected via ELISA and laser Doppler flow cytometry. However, the comparison of "periodontitis vs. healthy" was frequently examined in previous studies, but gingivitis, another important periodontal disease, was rarely included in research. In this context, the present study aimed to compare periodontitis and gingivitis patients vs. periodontally healthy subjects in terms of 27 markers in GCF. According to the results of the present study, statistically significantly higher IL-1 $\beta$, IL-6, MCP-1, IP-10 and VEGF levels were found in periodontitis patients compared to healthy subjects and also IL-15 levels were higher in healthy subjects. 
Table 4. Comparison of biochemical parameters

\begin{tabular}{|c|c|c|c|c|c|c|}
\hline \multirow{2}{*}{\multicolumn{2}{|c|}{$\begin{array}{l}\text { Biochemical } \\
\text { parameters }\end{array}$}} & \multicolumn{5}{|c|}{ Clinical parameters } \\
\hline & & \multirow{2}{*}{$\begin{array}{c}\text { PPD } \\
-0.290 *\end{array}$} & \multirow{2}{*}{$\begin{array}{c}\text { CAL } \\
-0.257^{*}\end{array}$} & \multirow{2}{*}{$\begin{array}{c}\text { GI } \\
-0.272 *\end{array}$} & \multirow{2}{*}{$\begin{array}{c}\text { PI } \\
-0.195\end{array}$} & \multirow{2}{*}{$\begin{array}{c}\text { ВОР } \\
-0.247^{*}\end{array}$} \\
\hline Eot & $r$ & & & & & \\
\hline & $p$ & 0.013 & 0.028 & 0.020 & 0.098 & 0.035 \\
\hline \multirow[t]{2}{*}{ FGF } & $r$ & -0.087 & -0.053 & -0.001 & 0.010 & 0.020 \\
\hline & $p$ & 0.465 & 0.656 & 0.992 & 0.935 & 0.864 \\
\hline \multirow[t]{2}{*}{ G-CSF } & $r$ & 0.004 & -0.010 & -0.012 & -0.019 & -0.014 \\
\hline & $p$ & 0.975 & 0.936 & 0.923 & 0.871 & 0.907 \\
\hline \multirow[t]{2}{*}{ GM-CSF } & $r$ & -0.195 & $-0.287 *$ & $-0.325^{* * *}$ & $-0.324^{* *}$ & $-0.339 * *$ \\
\hline & $p$ & 0.098 & 0.014 & 0.005 & 0.005 & 0.003 \\
\hline \multirow[t]{2}{*}{$\mathrm{IFN}-\gamma$} & $r$ & -0.069 & 0.027 & 0.012 & -0.041 & 0.020 \\
\hline & $p$ & 0.563 & 0.819 & 0.918 & 0.733 & 0.864 \\
\hline \multirow[t]{2}{*}{ IL-10 } & $r$ & -0.127 & -0.098 & -0.092 & -0.140 & -0.099 \\
\hline & $p$ & 0.284 & 0.409 & 0.436 & 0.238 & 0.407 \\
\hline \multirow[t]{2}{*}{ IL-12 } & $r$ & -0.158 & -0.132 & -0.137 & -0.172 & -0.119 \\
\hline & $p$ & 0.181 & 0.267 & 0.249 & 0.145 & 0.315 \\
\hline \multirow[t]{2}{*}{ IL-13 } & $r$ & -0.201 & $-0.237 *$ & -0.167 & -0.165 & -0.162 \\
\hline & $p$ & 0.088 & 0.044 & 0.158 & 0.163 & 0.171 \\
\hline \multirow[t]{2}{*}{ IL-15 } & $r$ & $-0.403 * *$ & $-0.422 * *$ & $-0.341^{* * *}$ & $-0.429 * *$ & $-0.357 * *$ \\
\hline & $p$ & 0.000 & 0.000 & 0.003 & 0.000 & 0.002 \\
\hline \multirow[t]{2}{*}{ IL-17 } & $r$ & -0.133 & 0.010 & 0.056 & 0.041 & 0.032 \\
\hline & $p$ & 0.260 & 0.934 & 0.641 & 0.730 & 0.785 \\
\hline \multirow[t]{2}{*}{ IL- $1 \beta$} & $r$ & $0.259 *$ & $0.287 *$ & $0.287 *$ & 0.207 & $0.298 *$ \\
\hline & $p$ & 0.027 & 0.014 & 0.014 & 0.078 & 0.011 \\
\hline \multirow[t]{2}{*}{ IL-1ra } & $r$ & -0.190 & -0.166 & -0.167 & -0.148 & -0.158 \\
\hline & $p$ & 0.108 & 0.161 & 0.159 & 0.212 & 0.182 \\
\hline \multirow[t]{2}{*}{ IL-2 } & $r$ & $-0.375^{* *}$ & $-0.334 * *$ & $-0.293 *$ & $-0.264 *$ & -0.300 ** \\
\hline & $p$ & 0.001 & 0.004 & 0.012 & 0.024 & 0.010 \\
\hline \multirow[t]{2}{*}{ IL-4 } & $r$ & -0.080 & 0.027 & 0.004 & 0.013 & 0.027 \\
\hline & $p$ & 0.503 & 0.822 & 0.976 & 0.911 & 0.819 \\
\hline
\end{tabular}

*Significant correlation $(p<0.05)$

$* *$ Strong significant correlation $(p<0.01)$

Cytokines and growth factors in GCF and also gingival tissues have frequently been investigated [13]. The most commonly used laboratory technique has so far been ELISA. In the ELISA method, the researcher can investigate only one biomarker with one sample. Cytokines are generally secreted synchronously with each other; therefore, it would be more meaningful to measure all cytokine levels comparatively in diseases with sophisticated immune mechanisms, especially in periodontal diseases. Multiplex bead systems have the capacity to measure nearly 500 biomarkers at once in a volume as small as 50 microliters [14]. After the first membrane-based multiplex immunoassay to profile the cytokines level in GCF

\begin{tabular}{|c|c|c|c|c|c|c|}
\hline \multirow{2}{*}{\multicolumn{2}{|c|}{$\begin{array}{l}\text { Biochemical } \\
\text { parameters }\end{array}$}} & \multicolumn{5}{|c|}{ Clinical parameters } \\
\hline & & \multirow{2}{*}{$\begin{array}{c}\text { PPD } \\
-0.043\end{array}$} & \multirow{2}{*}{$\begin{array}{c}\text { CAL } \\
-0.059\end{array}$} & \multirow{2}{*}{$\begin{array}{c}\text { GI } \\
-0.069\end{array}$} & \multirow{2}{*}{$\begin{array}{c}\text { PI } \\
-0.073\end{array}$} & \multirow{2}{*}{$\begin{array}{c}\text { ВОР } \\
-0.089\end{array}$} \\
\hline IL-5 & $r$ & & & & & \\
\hline & $p$ & 0.720 & 0.622 & 0.559 & 0.540 & 0.452 \\
\hline \multirow[t]{2}{*}{ IL-6 } & $r$ & 0.001 & 0.076 & -0.045 & 0.084 & -0.027 \\
\hline & $p$ & 0.996 & 0.520 & 0.708 & 0.482 & 0.821 \\
\hline \multirow[t]{2}{*}{ IL-7 } & $r$ & -0.107 & -0.022 & -0.076 & -0.056 & -0.070 \\
\hline & $p$ & 0.366 & 0.856 & 0.522 & 0.636 & 0.557 \\
\hline \multirow[t]{2}{*}{ IL-8 } & $r$ & -0.177 & -0.166 & -0.217 & -0.222 & $-0.235^{*}$ \\
\hline & $p$ & 0.134 & 0.161 & 0.065 & 0.059 & 0.045 \\
\hline \multirow[t]{2}{*}{ IL-9 } & $r$ & -0.220 & -0.065 & -0.038 & 0.032 & -0.074 \\
\hline & $p$ & 0.062 & 0.583 & 0.747 & 0.787 & 0.531 \\
\hline \multirow[t]{2}{*}{ IP-10 } & $r$ & $0.485^{* * *}$ & $0.348 * *$ & $0.351^{* *}$ & $0.337 * *$ & $0.375^{* * *}$ \\
\hline & $p$ & 0.000 & 0.003 & 0.002 & 0.004 & 0.001 \\
\hline \multirow[t]{2}{*}{ MCP-1 } & $r$ & -0.052 & 0.024 & 0.000 & 0.040 & 0.013 \\
\hline & $p$ & 0.660 & 0.838 & 1.000 & 0.739 & 0.912 \\
\hline \multirow[t]{2}{*}{ MIP- $1 \alpha$} & $r$ & -0.061 & 0.107 & 0.072 & 0.045 & 0.081 \\
\hline & $p$ & 0.609 & 0.367 & 0.547 & 0.708 & 0.498 \\
\hline \multirow[t]{2}{*}{ MIP-1 $\beta$} & $r$ & $-0.282 *$ & $-0.277^{*}$ & $-0.285^{*}$ & $-0.280 *$ & $-0.286 *$ \\
\hline & $p$ & 0.016 & 0.017 & 0.015 & 0.016 & 0.014 \\
\hline \multirow{2}{*}{$\begin{array}{c}\text { PDGF- } \\
\text { BB }\end{array}$} & $r$ & -0.202 & -0.166 & -0.188 & -0.141 & -0.201 \\
\hline & $p$ & 0.087 & 0.160 & 0.110 & 0.233 & 0.088 \\
\hline \multirow[t]{2}{*}{ RANTES } & $r$ & -.012 & 0.043 & 0.058 & -0.047 & 0.010 \\
\hline & $p$ & 0.919 & 0.717 & 0.628 & 0.695 & 0.930 \\
\hline \multirow[t]{2}{*}{ TNF- $\alpha$} & $r$ & -0.060 & -0.021 & 0.046 & 0.044 & 0.032 \\
\hline & $p$ & 0.613 & 0.857 & 0.697 & 0.715 & 0.786 \\
\hline \multirow[t]{2}{*}{ VEGF } & $r$ & -0.035 & 0.101 & 0.091 & 0.015 & 0.080 \\
\hline & $p$ & 0.769 & 0.395 & 0.444 & 0.902 & 0.499 \\
\hline
\end{tabular}

[15], dozens of studies have been conducted on this topic [16]. Multiple analysis of cytokines also provides the possibility of personal medicine. With a deep understanding of the importance of host tissue factors in periodontal disease in recent years, it is believed that personalized periodontal treatment methods will be investigated more frequently. Likewise, the current study contributes to the literature on this subject.

However, another point that should not be forgotten is the specific relationship between host response and microbial dental plaque. Studies have shown that specific bacteria in periodontitis such as Aggregatibacter actinomycetemcomitans are also present in periodontally healthy 
individuals [1]. In this context, the lack of microbial sampling and examination in the current study can be considered among the limitations. Longitudinal studies and cross-sectional studies are two types of studies frequently conducted in the field of periodontology. In a cross-sectional study, data are collected from a population at a certain time, while in a longitudinal study, data can be collected repeatedly from the same sample over a long period of time. Thus, it provides a better examination of the cause-effect relationship in longitudinal studies. In this context, the fact that the current study is cross-sectional can be considered as a limitation.

Biomarkers such as cytokines in GCF have been the subject of frequent research in the literature [7, 17]. Among these cytokines, probably the most important one, IL-1 $\beta$, is a proinflammatory cytokine that plays a key role in inflammation and immunity as well as increasing bone resorption [18]. It has been reported that IL-1 $\beta$ level is lower in healthy individuals compared to patients with periodontitis, and there is a decrease in IL- $1 \beta$ level after treatment in individuals with periodontitis [19]. It is accepted that IL- $1 \beta$ will increase with periodontal disease especially in periodontitis; in this regard, the results of the present study are in accordance with the current knowledge.

Interleukin 6 is a cytokine with proinflammatory and anti-inflammatory action. It plays a key role in many events such as B and T cell activation and differentiation, the occurrence of local acute phase events, and stimulation of osteoclast development. One study conducted by Offenbacher et al. [20] showed that patients with severe periodontitis had high levels of IL-1 $\beta$, IL-6, MCP-1, and prostaglandin $\mathrm{E} 2$, and these markers were associated with severe bleeding during probing. In addition, there are studies showing that IL-6 level decreased after non-surgical periodontal treatment $[18,21]$. According to the results of the present study, IL-6 level is high in individuals with periodontitis and it can be said that these data are compatible with the literature. Another important issue about IL-6 is that increased levels of the IL-6 molecule regulate the transition from acute to chronic inflammation and induce MCP-1 synthesis [22]. Macrophage chemotactic protein 1 stimulates the integrin synthesis required for chemotaxis during monocyte migration. It was reported that GCF contained higher levels of MCP-1 in individuals with periodontitis as compared to the healthy group and that the aforementioned level decreased after periodontal treatment [23]. In the study of Gunpinar et al., periodontitis patients and periodontally healthy individuals were compared in terms of MCP-1 levels in GCF and MCP-1 was found to be higher in patients with aggressive periodontitis [24]. Moreover, not only in vivo studies [24, 25] but also several in vitro studies [26, 27] showed that MCP-1 in gingival tissues was increased after inflammatory conditions such as periodontitis. Regarding MCP-1 levels in GCF, the present study was in accordance with the previous research.
IP-10 is a molecule that is secreted from various cells such as macrophages and endothelial cells. In addition to its chemoattractant properties for monocytes and macrophages, IP-10 controls the adhesion of T-cells to endothelial cells. According to the IP-10 data in the present study, it was observed that there was a statistically significant decrease in the periodontally healthy group compared to periodontitis patients; additionally, IP-10 was positively correlated with clinical parameters such as PPD, CAL, GI, PI, and BOP. Although Cairo et al. [28] did not find statistically significant differences between aggressive periodontitis and chronic periodontitis, Aldahlawi et al. [29] noted a statistically significantly higher level of IP-10 in saliva and serum, not GCF, in patients with periodontitis compared to periodontally healthy individuals. Moreover, Shimada et al. reported in their study that IP-10 levels were high in "bleeding on probing" (BOP) $(+)$ diseased areas [9]. The results of the present study were in accordance with the previous studies.

Interleukin 15 is a member of the IL-2 family of cytokines, and it was demonstrated that it inhibits osteoclast progenitor cell proliferation [30]. A study conducted by Johnson et al. [31] showed that there was a statistically significantly lower level of IL-15 in patients with periodontal disease compared to periodontally healthy subjects. Additionally, very few studies have been identified that address the relationship between IL-15 and periodontal health; therefore, the present study is one of the rare studies on this subject. In the present study, there was a statistically significantly lower level in the periodontally healthy group compared to the periodontitis group, and it is thought that IL-15 can be a parameter for periodontal health.

Profiling of cytokine and chemokine groups in GCF is frequently used in periodontology studies $[19,32]$. However, in these studies, aggressive or chronic periodontitis patients were frequently included in the periodontal disease groups and compared with periodontally healthy individuals. Moreover, the gingivitis group was not frequently included in the studies and could not be compared with the periodontally healthy group $[16,19]$. In this context, the present study is one of the rare studies comparing periodontitis and gingivitis patients with periodontally healthy individuals.

In conclusion, GCF is a good resource for identifying biomarkers in periodontal health and disease. Multiplex bead assays can be a useful tool in determining new biomarkers and for better understanding of the differences in cytokine profiles in periodontal disease and health. Within the limitations of this study, IL-1 $\beta$, IL-6, MCP-1, IP-10, and VEGF were identified as related to periodontal disease, and IL-15 was found to be a biomarker related to periodontal health.

\section{Acknowledgements}

The authors wish to thank Assoc. Prof. Yasemin Baskın and Research Assistant Mahdi Akbapour for their help in 
GCF analysis. This study was supported by a grant from the Gaziantep University Research Foundation (number DHF 13.05), Gaziantep, TURKEY. All procedures performed in studies involving human participants were in accordance with the ethical standards of the Clinical Ethic Committee of Gaziantep University (16.04.2013/168) and with the 1964 Helsinki declaration and its later amendments or comparable ethical standards. Written/verbal informed consent was obtained from the patients and the parents of the patients who participated in this study.

\section{The authors declare no conflict of interest.}

\section{References}

1. Kantarci A, Hasturk H, Van Dyke TE, et al. (2006): Host-mediated resolution of inflammation in periodontal diseases. Periodontol 2000 40: 144-163.

2. Garlet GP (2010): Critical reviews in oral biology \& medicine: Destructive and protective roles of cytokines in periodontitis: A re-appraisal from host defense and tissue destruction viewpoints. J Dent Res 89: 1349-1363.

3. Berezow AB, Darveau RP (2011): Microbial shift and periodontitis. Periodontol 2000 55: 36-47.

4. Giannobile WV (2008): Host-response therapeutics for periodontal diseases. J Periodontol 79: 1592-1600.

5. Hajishengallis G (2014): Immunomicrobial pathogenesis of periodontitis: keystones, pathobionts, and host response. Trends Immunol 35: 3-11.

6. Barrientos S, Brem H, Stojadinovic O, Tomic-Canic M (2014): Clinical application of growth factors and cytokines in wound healing. Wound Repair Regen 22: 569-578.

7. Stadler AF, Angst PDM, Arce RM, et al. (2016): Gingival crevicular fluid levels of cytokines/chemokines in chronic periodontitis: a meta-analysis. J Clin Periodontol 43: 727-745.

8. Teles R, Sakellari D, Teles F, et al. (2010): Relationships among gingival crevicular fluid biomarkers, clinical parameters of periodontal disease, and the subgingival microbiota. J Periodontol 81: 89-98.

9. Shimada Y, Tabeta K, Sugita N, Yoshie H (2013): Profiling biomarkers in gingival crevicular fluid using multiplex bead immunoassay. Arch Oral Biol 58: 724-730.

10. Duarte PM, Bezerra JP, Miranda TS, et al. (2014): Local levels of inflammatory mediators in uncontrolled type 2 diabetic subjects with chronic periodontitis. J Clin Periodontol 41: 11-18.

11. Armitage GC (1999): Development of a classification system for periodontal diseases and conditions. Ann Periodontol 4: 1-6.

12. Caton JG, Armitage G, Berglundh T, et al. (2018): A new classification scheme for periodontal and peri-implant diseases and conditions - Introduction and key changes from the 1999 classification. J Periodontol 89: S1-S8.

13. Delima AJ, Van Dyke TE (2003): Origin and function of the cellular components in gingival crevice fluid. Periodontol 200031 : 55-76.

14. Wang CC, Huang RP, Sommer M, et al. (2002): Array-based multiplexed screening and quantitation of human cytokines and chemokines. J Proteome Res 1: 337-343.

15. Sakai A, Ohshima M, Sugano N, et al (2006): Profiling the cytokines in gingival crevicular fluid using a cytokine antibody array. J Periodontol 77: 856-864.
16. Bostanci N, Belibasakis GN (2018): Gingival crevicular fluid and its immune mediators in the proteomic era. Periodontol 200076 : 68-84.

17. Teles RP, Gursky LC, Faveri M, et al. (2010): Relationships between subgingival microbiota and GCF biomarkers in generalized aggressive periodontitis. J Clin Periodontol 37: 313-323.

18. Liu YCG, Lerner UH, Teng YTA (2010): Cytokine responses against periodontal infection: protective and destructive roles. Periodontol 2000 52: 163-206.

19. Thunell DH, Tymkiw KD, Johnson GK, et al. (2010): A multiplex immunoassay demonstrates reductions in gingival crevicular fluid cytokines following initial periodontal therapy. J Periodontal Res 45: 148-152.

20. Offenbacher S, Barros SP, Singer RE, et al. (2007): Periodontal disease at the biofilm-gingival interface. J Periodontol 78: 19111925.

21. Preshaw PM, Taylor JJ (2011): How has research into cytokine interactions and their role in driving immune responses impacted our understanding of periodontitis? J Clin Periodontol 38 Suppl 1: 60-84.

22. Kaplanski G, Marin V, Montero-Julian F, et al. (2003): IL-6: A regulator of the transition from neutrophil to monocyte recruitment during inflammation. Trends Immunol 24: 25-29.

23. Gupta M, Chaturvedi R, Jain A (2013): Role of monocyte chemoattractant protein-1 (MCP-1) as an immune-diagnostic biomarker in the pathogenesis of chronic periodontal disease. Cytokine 61: 892-897.

24. Gunpinar S, Alptekin NO, Dundar N (2017): Gingival crevicular fluid levels of monocyte chemoattractant protein-1 in patients with aggressive periodontitis. Oral Dis 23: 763-769.

25. Martins ES, César-Neto JB, Albuquerque-Souza E, et al. (2019): One-year follow-up of the immune profile in serum and selected sites of generalized and localized aggressive periodontitis. Cytokine 116: 27-37.

26. Garlet GP, Martins W, Ferreira BR, et al. (2003): Patterns of chemokines and chemokine receptors expression in different forms of human periodontal disease. J Periodontal Res 38: 210-217.

27. Pradeep AR, Daisy H, Hadge P, et al. (2009): Correlation of gingival crevicular fluid interleukin-18 and monocyte chemoattractant protein-1 levels in periodontal health and disease. J Periodontol 80: 1454-1461.

28. Cairo F, Nieri M, Gori AM, et al. (2010): Markers of systemic inflammation in periodontal patients: Chronic versus aggressive periodontitis. An explorative cross-sectional study. Eur J Oral Implantol 3: 147-153.

29. Aldahlawi S, Youssef AR, Shahabuddin S (2018): Evaluation of chemokine CXCL10 in human gingival crevicular fluid, saliva, and serum as periodontitis biomarker. J Inflamm Res 11: 389-396.

30. Saeed S, Revell PA (2001): Production and distribution of interleukin 15 and its receptors (IL-15Ralpha and IL-R2beta) in the implant interface tissues obtained during revision of failed total joint replacement. Int J Exp Pathol 82: 201-209.

31. Johnson RB, Serio FG (2007): The contribution of interleukin-13 and -15 to the cytokine network within normal and diseased gingiva. J Periodontol 78: 691-695.

32. Zein Elabdeen HR, Mustafa M, Ali R, Bolstad AI (2017): Cytokine profile in gingival crevicular fluid and plasma of patients with aggressive periodontitis. Acta Odontol Scand 75: 616-622. 